\title{
Acute Pain Management in Opioid Dependent Patients
}

\author{
Vivek Mehta and Richard Langford \\ Consultants in Pain Medicine and Anaesthesia, \\ Pain and Anaesthesia Research Centre, St Bartholomew's Hospital. \\ Barts and The London NHS Trust, London, UK.
}

\section{SUMMARY POINTS:}

- Acute pain management remains a challenge in opioid dependent patients, and it has been recognised that these patients are commonly under-treated.

- Chronic opioid exposure leads to widespread adaptations both at cellular and synaptic level.

- Physical dependence is a neuropharmacological phenomenon as a result of neuroadaptation and neuroplasticity, in contrast to addiction that is both neuropharmacological and behavioural.

- While providing the patient's pre-existing opioid requirement, the acute pain episode should be managed using additional multimodal analgesia: non-opioid medications in combination with local anaesthetic techniques and as required, short-acting opioid titrated to effect.

- Patients on long term buprenorphine and methadone with acute pain episode should be continued with their maintenance therapy and an additional short-acting opioid analgesic titrated to achieve therapeutic effect.

\section{Introduction}

Opioid use has been steadily gaining acceptance as an appropriate and effective method of pain management in persistent non-cancer pain in both Europe and the USA supported by evidence based guidelines ${ }^{1,2}$. Besides their therapeutic indication in cancer and noncancer pain, opioids are associated with illicit recreational use and also provide a substantial component of treatment in maintenance programmes for addicts. Long-term opioid prescriptions are increasing, with approximately $70 \%$ of patients attending a tertiary hospital chronic pain clinic on some form of opioid (including codeine). Given the increasing prevalence of chronic pain as a result of our ageing population, the use of prescribed opioid analgesics is likely to increase further. The British Crime Survey 2005/06 commissioned by the Home Office revealed a $0.1 \%$ incidence of heroin and methadone usage in the population aged 16-59 years ${ }^{3}$. This level has been stable over the last ten years, with estimated total user numbers of 39,000 and 33,000 for the two drugs respectively.

Chronic opioid intake (whether recreational or long term therapeutic prescription) may result in physical dependence, manifested by a drug specific withdrawal syndrome that can be produced by abrupt cessation. Though these individuals show dependence, they are not necessarily addicts.
Defined by a Joint Consensus Statement of the American Academy of Pain Medicine, the American Pain Society, and the American Society of Addiction Medicine, physical dependence is a state of adaptation that is manifested by a drug class specific withdrawal syndrome that can be produced by abrupt cessation, rapid dose reduction, decreasing blood level, and/or administration of an antagonist. Physical dependence is a neuropharmacological phenomenon as a result of neuroadaptation and neuroplasticity.

By contrast, addiction is both a neuropharmacological and a behavioural phenomenon. Addiction is a primary, chronic, neurobiological disease, with genetic, psychosocial, and environmental factors influencing its development and manifestations. It is characterised by behaviour that includes one or more of the following: impaired control over drug use, compulsive use, continued use despite harm, and drug craving.

Acute pain management remains a challenge in opioid dependent patients, and it has been recognised that these patients are commonly under-treated ${ }^{4}$. 


\section{Pathophysiology of opioid dependence}

Chronic opioid exposure leads to widespread adaptations at cellular and synaptic level, thought to be responsible for the phenomena of tolerance and dependence. These neuroadaptations may develop at multiple levels including a) the $\mu$-opioid receptor itself, b) the neurons that express these receptors $\mathrm{c}$ ) the neural and neuronal-glial networks which interact with these neurons and d) synaptic plasticity and learning in these opioid sensitive nerve networks.

a) Receptor level: Though several potential mechanisms may be responsible, the in-vitro studies show that there is a loss in coupling of $\mu$-opioid receptor to a major cellular effector, the G-protein regulated inwardly rectifying $\mathrm{K}+$ channel ${ }^{5}$. Chronic opioid exposure results in desensitisation and internalisation of the receptor. Internalisation leads to removal of receptors from the external membrane surface predominantly from G-protein coupled receptor kinase (GRK) and endosomal mechanisms. It is speculated that variation in the propensity of different opioid agonists to induce receptor internalisation is responsible for their likelihood of inducing addiction or opioid dependence. As shown by in-vitro studies, it may be possible that agonists employed therapeutically in dependency management such as methadone, D-ala-methionine ekephalin glyol (DAMGO) and buprenorphine, demonstrate beneficial effects on adaptive processes as they may induce greater internalisation than morphine $e^{6,7,8}$.

b) Cellular adaptations: The cAMP hypothesis remains the most thoroughly studied mechanism in support of the cellular adaptations with respect to tolerance and withdrawal. At the cellular level, opioids decrease adenyl cyclase enzyme production of intracellular cAMP. Chronic exposure to opioids induces adenyl cyclase hence slowly increasing the intracellular cAMP to 'normal' levels s, $^{90}$. Activation of the cyclic AMP cascade results in activation of phosphokinase $\mathrm{A}$, and release of transmitters including GABA and glutamate. The concentration of cyclic AMP increases in the extracellular space, where it is metabolised by phosphodiesterases to AMP and rapidly converted to adenosine by ecto-nucleotidases. If long-term opioid therapy is suddenly discontinued, the falling adenyl cyclase enzyme levels precipitate a withdrawal syndrome. This however remains a simplistic explanation, as in vitro studies have shown that distinct mechanisms are responsible for the differential regulation of each of the nine isozymes of adenyl cyclase. Further, the adaptations are complicated by

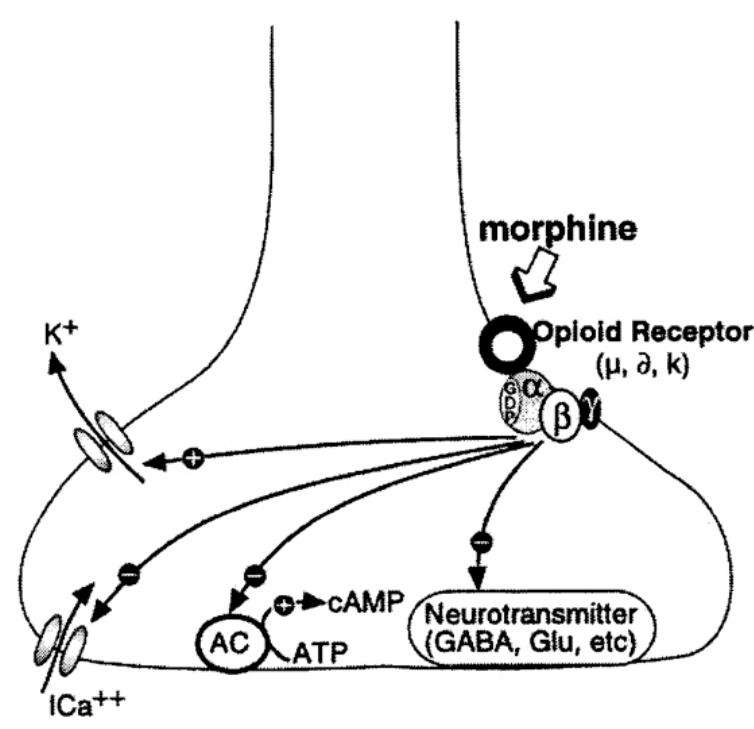

ACUTE OPIOID

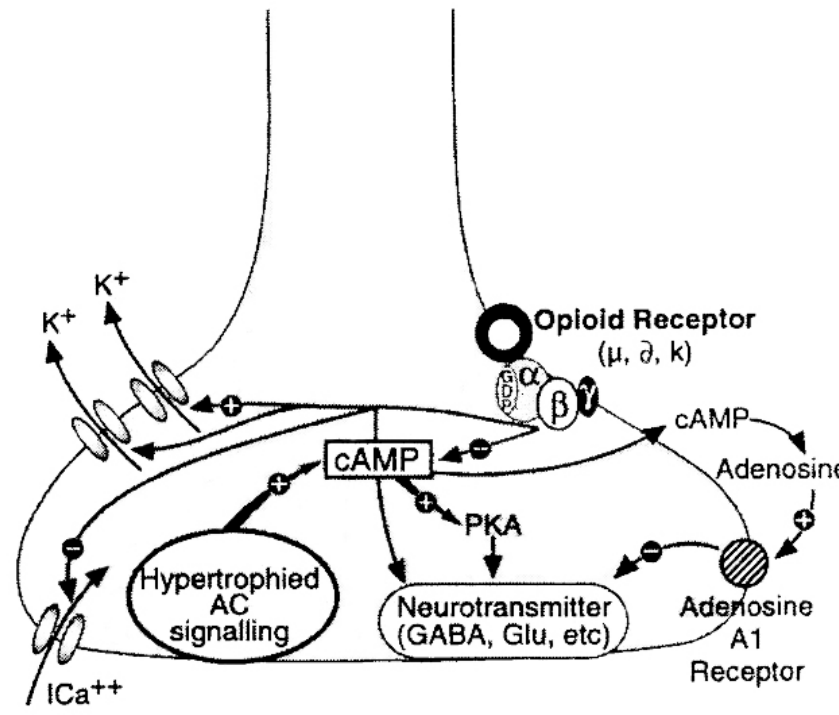

Counter adaptations AFTER CHRONIC MORPHINE

(Inhibition of new or hypertrophied transduction pathway)

Morphine's cellular actions in the 'opioid dependant' situation

Morphine's cellular actions in the 'opioid naïve' situation 
a range of protein signalling complexes that include kinases, phosphatases and phosphodiesterases, and may exhibit an individual variability depending upon the cell types and the cellular compartments ${ }^{11}$.

c) Systemic adaptations: Systemic adaptations in opioid sensitive neuronal and neuroglial networks can also develop. This is usually manifested by a change in electrical activity in a component of a neuronal network inducing adaptations in other neurons and synapses throughout this or other networks. For example, despite no direct action of $\mu$-opioid agonists on dopaminergic neurons, it has been suggested that widespread adaptations in this particular network may be responsible for symptoms in addiction ${ }^{12}$.

d) Synaptic plasticity: Synaptic learning and memory is influenced by chronic opioid exposure. This can be attributed to both long-term potentiation and depression; two processes involved in neural memory. These processes involve glutamate mediated changes including increased AMPA receptor mediated neurotransmission in synapses subsequently affecting the synaptic strength. Such changes are well established at many opioid sensitive GABAergic synapses, but are also heavily influenced by the cellular and systemic adaptations described earlier.

\section{Management of acute pain in patients on long-term opioid agonists}

Opioid dependent patients may be either drug abusers, or patients on chronic opioid analgesic therapy. Effective management must combine analgesic treatment and prevention of withdrawal reactions and complications. Given the variability in composition of recreational drugs, it can be difficult to estimate the morphine equivalent dose, and clearly there are risks with errors either way. Inadequate opioid provision may both under-treat the pain and also precipitate withdrawal reactions. It is important that personnel competent in opioid dose adjustment ensure that baseline opioid requirements are met, and additional analgesia provided to manage the acute pain ${ }^{13}$.

If the patient is on pure $\mu$ agonist drugs (morphine, codeine, fentanyl, hydromorphone) then they should be continued on the pre-existing medication or equivalent dose of another opioid. As for any patient, their new onset, acute episode analgesic requirements should be met using multimodal analgesia: non-opioid medications including local anaesthetic techniques and as required, short-acting opioid titrated to effect ${ }^{13}$. Paracetamol and NSAIDs being devoid of central depressant or mood altering effects are ideal, and preferably used in combination. The additional short-acting opioid can subsequently be down-titrated, as the acute episode subsides.

Switching to a new opioid, (opioid rotation), was pioneered in palliative care medicine for patients who developed tolerance and/ or unacceptable side effects. The substituted opioid may achieve both improved pain relief at a lower than equianalgesic dose, and fewer side effects. However, the degree of cross-tolerance of different opioids is unpredictable and hence caution is essential in selecting the dose ${ }^{14}$. The published dose equivalence tables provide guidance, but it is wise to seek expert advice. It is common practice to start initially at around two thirds of the calculated equivalent dosage and then titrate according to the response. Further, it is important to recognise the analgesic duration of the opioid medication whilst switching. Switching from long-acting opioids to intermittent doses of a short-acting opioid medication, may trigger withdrawal episodes in dependent patients, who require maintenance above a certain plasma level of opioid, warranting either frequent repeat doses or a continuous infusion.

\section{Patients on buprenorphine}

The reformulation of buprenorphine as a sustained release, transdermal matrix patch for the treatment of chronic pain has generated renewed interest in the pharmacological properties of this opioid analgesic, initially considered to have agonist-antagonist activity. In its transdermal preparation, it takes nine days to reach steady state plasma concentration, and a reservoir of buprenorphine accumulates in the skin, such that buprenorphine continues to be absorbed from the skin for around twelve hours, after removal of the patch. Transdermal buprenorphine has been a cause for concern in acute pain management, as it is feared that buprenorphine's high affinity for the $\mu$ receptor may impede the actions of additional opioid agonist analgesics. Limited clinical experience, expert opinion, pharmacologic principles and published recommendations suggest that buprenorphine does not antagonise the analgesic effectiveness of other opioids. Nevertheless, it is a potentially unpredictable situation, and the analgesic efficacy, level of consciousness and respiration should be frequently monitored. If 'reversal' of buprenorphine is required, higher and more frequent doses of an antagonist, such as naloxone, may be required ${ }^{15}$.

Various acute pain treatment strategies for patients on long term buprenorphine have been described:

1. Ideally, buprenorphine maintenance therapy should be continued and a short-acting opioid analgesic titrated to achieve therapeutic effect ${ }^{16,17}$.

2. Another option is to discontinue buprenorphine therapy, well ahead of elective surgery, and substitute a pure opioid agonist analgesic ${ }^{16,17,18}$. Following resolution of the acute pain episode, one could consider switching back to maintenance therapy with buprenorphine ${ }^{17}$. 
3. Alternatively, one could switch to methadone, (which binds less tightly to the $\mu$ receptor) at a dose sufficient to prevent acute withdrawal in most patients, such as 30 to $40 \mathrm{mg} /$ day $^{19}$. If opioid withdrawal still persists, methadone doses can be increased in 5 to $10 \mathrm{mg}$ increments ${ }^{20}$. However methadone has less predictable pharmacokinetics and is not generally used for acute pain management. Hence this method may be more appropriate in patients on buprenorphine maintenance programmes who present with acute pain, for whom there is then the option to be continued on methadone after the acute pain episode has subsided.

\section{Patients on maintenance programmes}

Maintenance therapy for opioid addiction cannot be relied upon to provide analgesia during an episode of acute pain. Methadone and buprenorphine, are commonly administered every $24-48$ hours and have a duration of action for analgesia of 4 to 8 hours, which is substantially shorter than that for suppression of opioid withdrawal. Hence the period of even partial pain relief with these maintenance medication doses is small and the need as stated above for additional acute pain treatment measures, which can include short-acting potent opioid analgesics ${ }^{21}$.

Albeit unsubstantiated, there have been concerns that the usage of opioids in patients on long-term opioid maintenance therapy for treatment of addiction, may trigger a relapse. However, there is no evidence that exposure to opioid analgesics in the presence of acute pain increases rates of relapse in such patients. A small retrospective study of patients enrolled in maintenance methadone programs that received opioid analgesics after surgery did not find a difference in relapse indicators compared with matched patients receiving maintenance methadone therapy ${ }^{22}$. Similarly, no evidence of relapse was seen in six patients receiving methadone maintenance therapy who were treated with opioid analgesics for cancer-related pain ${ }^{23}$.

Acute pain management for the patient receiving maintenance methadone therapy should follow the general recommendations, which include using opioid analgesics, when indicated, in addition to the patient's daily methadone maintenance dose. If oral intake is not feasible, an equivalent parenteral opioid dose is given, or methadone itself can be given parenterally in some countries (intramuscular or subcutaneous methadone dosage given as half to two thirds the maintenance dose divided into 2 to 4 equal doses), but not in the $\mathrm{UK}$, where it is not available ${ }^{24}$.

\section{Conclusion}

Acute pain management in opioid dependent patients remains a challenge for anaesthetists and acute pain services. The pre-existing opioid requirement is provided as a baseline, to which is added, appropriate multimodal analgesia. If necessary, and as for any patient, short-acting opioids are provided for prn usage, and discontinued once the acute episode subsides. Guidance with respect to sustained release buprenorphine is limited, but these patients appear to benefit equally from a simultaneous short-acting opioid analgesic titrated to response. If elective surgery is planned, then an alternative strategy may be to discontinue the buprenorphine and substitute a pure $\mu$ receptor opioid.

\section{REFERENCES}

1. Zenz M, Strumph M, Tryba M. Long term oral opioid therapy in patients with chronic non-malignant pain. Journal of Pain and Symptom management 1992; 7(2): 69-77.

2. Schug S, Merry A, Acland R. Treatment principles for the use of opioids in pain of non-malignant origin. Drugs 1991; 42(2): 228-232.

3. Walker A, Kershaw C, Nicholas S. Crime in England and Wales 2005/06. Home Office Statistical Bulletin. www.homeoffice.gov. $\mathrm{uk} / \mathrm{rds}$

4. Pallasch TJ. Anaesthetic management of the chemically dependent patient. Anesthesia Progress 1992; 39: 157-61.

5. Connor M, Osborne PB, Christie MJ. Mu-opioid receptor desensitization: is morphine different? Br J Pharmacol 2004; 143: 685-96.

6. Keith DE, Murray SR, Zaki PA, Chu PC, Lissin DV, Kang L, et al. Morphine activates opioid receptors without causing their rapid internalization. J Biol Chem 1996; 271: 19021-4.

7. Whistler JL, Chuang HH, Chu P, Jan LY, von Zastrow M. Functional dissociation of mu opioid receptor signalling and endocytosis; implications for the biology of opiate tolerance and addiction. Neuron 1999; 23: 737-46.

8. Borgland SL, Connor M, Osborne PB, Furness JB, Christie MJ. Opioid agonists have different efficacy profiles for $\mathrm{G}$ protein activation, rapid desensitization, and endocytosis of mu-opioid receptors. J Biol Chem 2003; 278: 18776-84.

9. Avidor-Reiss T, Nevo I, Pfeuffer T, Vogel Z. Chronic opioid treatment induces adenylyl cyclase $\mathrm{V}$ superactivation. J Biol Chem 1996; 271:21309-21315.

10. Avidor-Reiss T, Nevo I, Saya D, Bayewitch M, Vogel Z. Opiate induced adenylyl cyclase superactivation is isoenzyme -specific. J Biol Chem 1997; 272: 5040-5047.

11. Wong W, Scott JD. AKAP signalling complexes: focal points in space and time. Nature Rev Mol Cell Biol 2004; 5: 959-70.

12. Russo SJ, Bolanos CA, Theobald DE, DeCarolis NA, Renthal W, Kumar A, et al. IRS2-Akt pathway in midbrain dopamine neurons regulates behavioral and cellular responses to opiates. Nature Neurosci 2007; 10: 93-9. 
13. Mehta V, Langford RM. Acute pain management in opioid dependent patients. Anaesthesia 2006; 61: 269-76.

14. Savage SR. Principles of pain treatment in the addicted patient. In: Graham AW, Schultz TK (eds). Principles of Addiction Medicine. 2nd edn. Chevy Chase, MD: American Society of Addiction Medicine, Inc. 1998, 919-944.

15. Mehta V, Phillips JP, Wantman AC, Ratcliffe SH, van Raders PA, Langford RM. Investigation of buprenorphine induced respiratory depression in anaesthetised patients and its reversibility. British J Anaesthesia 2005; 94: 399-400.

16. Mitra S, Sinatra RS. Perioperative management of acute pain in the opioid-dependent patient. Anesthesiology 2004;101: 212-27.

17. Center for Substance Abuse Treatment. Clinical Guideline for the Use of Buprenorphine in the Treatment of Opioid Addiction. Treatment Improvement Protocol (TIP) Series 40. DHHS publication no. (SMA) 04-3939. Rockville, MD: Substance Abuse and Mental Health Services Administration; 2004.

18. McCance-Katz EF. Office-based buprenorphine treatment for opioid-dependent patients. Harv Rev Psychiatry 2004;12:32138.
19. Fultz JM, Senay EC. Guidelines for the management of hospitalized narcotics addicts. Ann Intern Med 1975;82:815-8.

20. Center for Substance Abuse Treatment. State Methadone Treatment Guidelines. Treatment Improvement Protocol (TIP) Series 1. DHHS publication no. (SMA) 93-1991. Rockville, MD: Substance Abuse and Mental Health Services Administration; 1993.

21. Alford DP, Compton P, Samet JH. Acute Pain Management for Patients Receiving Maintenance Methadone or Buprenorphine Therapy. Annals of Internal Medicine 2006;144: 127-34.

22. Kantor TG, Cantor R, Tom E. A study of hospitalized surgical patients on methadone maintenance. Drug Alcohol Depend 1980;6:163-73.

23. Manfredi PL, Gonzales GR, Cheville AL, Kornick C, Payne R. Methadone analgesia in cancer pain patients on chronic methadone maintenance therapy. J Pain Symptom Manage 2001;21:169-74.

24. Fishman SM, Wilsey B, Mahajan G, Molina P. Methadone reincarnated: novel clinical applications with related concerns. Pain Med 2002; 3:339-48. 